\title{
Transport of Twelve Coumarins from Angelicae Pubescentis Radix across a MDCK-pHaMDR Cell Monolayer-An in Vitro Model for Blood-Brain Barrier Permeability
}

\author{
Yan-Fang Yang, Wei Xu, Wei Song, Min Ye and Xiu-Wei Yang * \\ State Key Laboratory of Natural and Biomimetic Drugs, Department of Natural Medicines, \\ School of Pharmaceutical Sciences, Peking University Health Science Center, Peking University, No. 38, \\ Xueyuan Road, Haidian District, Beijing 100191, China; E-Mails: yangyanfang@bjmu.edu.cn (Y.-F.Y.); \\ high-xu@163.com (W.X.); sw-yy1990@163.com (W.S.); yemin@bjmu.edu.cn (M.Y.) \\ * Author to whom correspondence should be addressed; E-Mail: xwyang@bjmu.edu.cn; \\ Tel.: +86-10-8280-1569; Fax: +86-10-8280-2724.
}

Academic Editor: Pascal Richomme

Received: 16 May 2015 / Accepted: 23 June 2015 / Published: 25 June 2015

\begin{abstract}
Angelicae Pubescentis Radix (APR), a widely used traditional Chinese medicine, is reported to have central nervous system activities. The purpose of this study was to characterize the blood-brain barrier permeability of twelve coumarins from APR including umbelliferone (1), osthol (2), scopoletin (3), peucedanol (4), ulopterol (5), angepubebisin (6), psoralen (7), xanthotoxin (8), bergapten (9), isoimperatorin (10), columbianadin (11), and columbianetin acetate (12) with an in vitro model using a MDCK-pHaMDR cell monolayer. The cell monolayer was validated to be suitable for the permeation experiments. The samples' transports were analyzed by high performance liquid chromatography and their apparent permeability coefficients $\left(P_{a p p}\right)$ were calculated. According to the $P_{a p p}$ value, most coumarins could be characterized as well-absorbed compounds except for 4, 10 and 11 which were moderately absorbed ones, in concentration-dependent and time-dependent manners. The results of P-glycoprotein (P-gp) inhibitor (verapamil) experiments showed that the transport of coumarin 4 was affected by the transport protein P-gp. Sigmoid functions between permeability $\log \left(P_{\text {app } \mathrm{AP}-\mathrm{BL}} * \mathrm{MW}^{0.5}\right)$ and $\log \mathrm{D}($ at $\mathrm{pH} 7.4)$ were established to analyze the structure-activity relationship of coumarins. The results provide useful information for discovering the substance basis for the central nervous system activities of APR, and predicting the permeability of other coumarins through BBB.
\end{abstract}


Keywords: Angelicae Pubescentis Radix; coumarins; MDCK-pHaMDR cells; blood brain barrier; permeability; structure-permeability relationship

\section{Introduction}

Phytochemicals have been used as promising therapeutic agents for encephalopathy in recent years. Angelicae Pubescentis Radix (APR, the roots of Angelica pubescens Maxim. f. biserrata Shan et Yuan), known as Duhuo in Chinese, is one of the most widely used traditional Chinese medicines. Pharmacological studies indicate that APR possesses anti-inflammatory [1], analgesic [2], anticancer [3] and platelet aggregation inhibitory [4] activities. Other studies show that APR also has central nervous system (CNS) activities [5] of inhibiting the apoptosis of brain cells [6], protecting from $\mathrm{H}_{2} \mathrm{O}_{2}$-induced SH-SY5Y cells injury [7], and being effective in treating Alzheimer's disease (AD) [8]. Coumarins are the main active components of APR, and more than 60 coumarins have isolated and identified from APR [9]. Previous studies have reported that many of these coumarins have biological CNS activities. For instance, scopoletin has anti cholinergic- and age-impaired memory ameliorative activities [10,11]; osthole possesses the ability to protect cortical neurons and SH-SY5Y cells against $\beta$-amyloid peptide (A $\beta)$ injury [12] and traumatic brain injury [13]; isoimperatorin [14], psoralen [15] and xanthotoxin [16,17] can inhibit both acetylcholinesterase activity for the treatment of $\mathrm{AD}$ and brain monoamine oxidase activity for the treatment of affective disorders. The above studies suggest that coumarins are the prime ingredients contributing to the CNS activity of APR. The intestinal absorptions of main coumarins from APR, such as umbelliferone, osthol, columbianadin, columbianetin acetate, psoralen, bergapten, xanthotoxin and isoimperatorin, have been studied with human colon adenocarcinoma cell line (Caco-2) cell monolayer in our group, and all of them are defined as well or moderately intestinal absorbed compounds [18-20]. Therefore, it is necessary to study the penetration abilities of the main coumarins of APR through the blood-brain barrier (BBB) for accessing its CNS pharmacological activities.

$\mathrm{BBB}$ permeability is one of the key determinants for CNS exposure and the kinetics evaluation of drugs [21]. BBB is a strong barrier between the blood and brain parenchyma, providing the stable microenvironment that is critical for complex neural function and protecting the CNS from chemical insult and damage [22]. Due to the high complexity of both passive penetration and active transport processes, cell cultures are the favored tools for BBB drug penetration prediction. Among them, the multidrug resistant Madin-Darby Canine kidney (MDCK-MDR1) cell line has been proved to be a simple, quick and proper surrogate BBB model on the basis of the electron microscopical morphology, transepithelial electrical resistance (TEER), transport functionality and P-glycoprotein (P-gp) function [23,24]. Under the cell culture conditions, MDCK-MDR1 cells form a monolayer after eight days and exhibit high TEER because of the tight junction expression [23]. MDCK-MDR1 cell monolayer has been used to study the BBB permeability of different active constituents from natural medicines, such as ginkgolides [25], decursin and decursinol angelate [26].

In this paper, MDCK-pHaMDR cell line, derived from the parental MDCK cell line after infection with the MDR1 virus produced by the cell line PA-12-MDR1/A1 [27], was obtained from National Institutes of Health (NIH, Bethesda, MD, USA), and utilized to study the BBB penetration of twelve coumarins 
from APR (chemical structures shown in Figure 1), along with the time- and concentration-dependent absorption. The samples were analyzed by high performance liquid chromatography (HPLC) and the transport parameter apparent permeability coefficient $\left(P_{\text {app }}\right)$ was calculated. The aim is to discover the substance basis for the CNS activities of APR and the possible transport mechanisms of coumarins through $\mathrm{BBB}$, together with the BBB permeability prediction of coumarins.

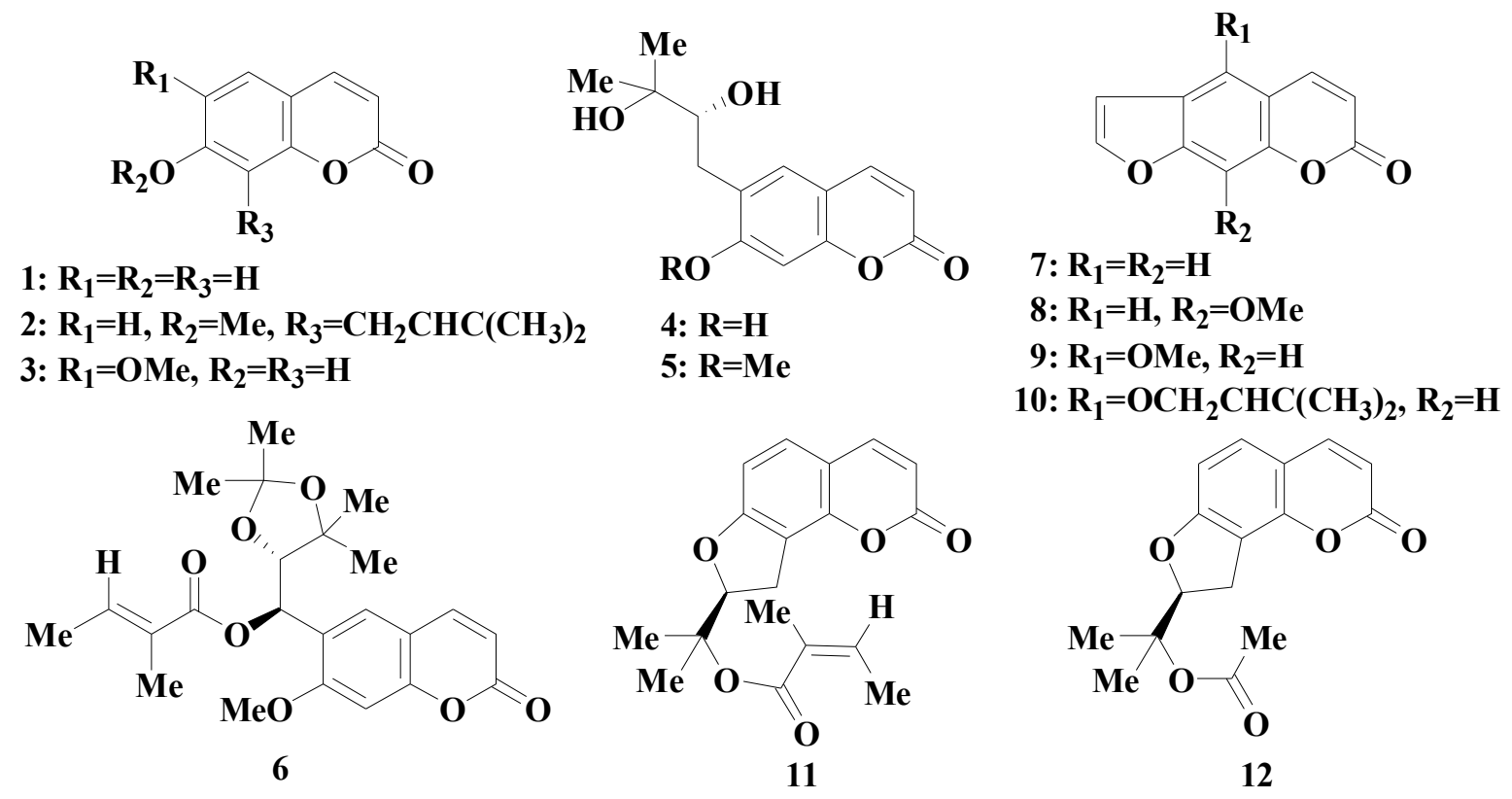

Figure 1. Chemical structures of coumarins from Angelicae Pubescentis Radix: umbelliferone (1), osthol (2), scopoletin (3), peucedanol (4), ulopterol (5), angepubebisin (6), psoralen (7), xanthotoxin (8), bergapten (9), isoimperatorin (10), columbianadin (11) and columbianetin acetate (12).

\section{Results and Discussion}

\subsection{Validation of the MDCK-pHaMDR Cell System}

The applicability of MDCK-pHaMDR cell monolayer as in vitro BBB model was validated. MTT assays showed that all coumarins at the maximum test concentration of $125 \mu \mathrm{M}$ exerted no significant influence on cell viability.

As shown in Table 1, the TEERs of test cell monolayer were all above $1000 \Omega \cdot \mathrm{cm}^{2}$ in accord with the reported values [26]. Caffeine and atenolol (as the well- and poor-transported marker by passive diffusion) were run from the apical side (AP) to basolateral side (BL) across the MDCK-pHaMDR cell monolayer, and $P_{\text {app }}$ values were calculated as $4.36 \times 10^{-5} \mathrm{~cm} / \mathrm{s}$ and $7.61 \times 10^{-7} \mathrm{~cm} / \mathrm{s}$, respectively. The results were comparable to reported values [26] and the applicability of the cell monolayer as in vitro BBB model was verified. 
Table 1. The bidirectional $P_{a p p}$ values of twelve coumarins in the MDCK-pHaMDR cell monolayer.

\begin{tabular}{cccccc}
\hline Compound & $\begin{array}{c}\boldsymbol{P}_{a p p \text { AP-BL }} \\
\left(\times \mathbf{1 0}^{-\mathbf{6}} \mathbf{c m} / \mathbf{s}\right)\end{array}$ & $\begin{array}{c}\boldsymbol{P}_{a p p} \text { BL-AP } \\
\left(\times \mathbf{1 0}^{-\mathbf{6}} \mathbf{c m} / \mathbf{s}\right)\end{array}$ & $\begin{array}{c}\boldsymbol{P}_{a p p \text { BL-AP }} / \\
\boldsymbol{P}_{a p p \text { AP-BL }}\end{array}$ & $\begin{array}{c}\text { LogD } \\
(\mathbf{p H} 7.4)\end{array}$ & $\begin{array}{c}\text { TEER } \\
\left(\mathbf{\Omega} \cdot \mathbf{c m}^{\mathbf{2}}\right)\end{array}$ \\
\hline $\mathbf{1}$ & $35.86 \pm 2.28$ & $32.79 \pm 1.25$ & 0.91 & 1.44 & $1321 \pm 129$ \\
$\mathbf{2}$ & $23.79 \pm 1.44$ & $20.37 \pm 3.04$ & 0.86 & 4.08 & $1548 \pm 104$ \\
$\mathbf{3}$ & $37.10 \pm 1.97$ & $27.38 \pm 3.51$ & 0.96 & 1.65 & $1586 \pm 194$ \\
$\mathbf{4}$ & $9.91 \pm 0.21$ & $18.97 \pm 0.38$ & 1.91 & 0.63 & $1595 \pm 93$ \\
$\mathbf{5}$ & $22.36 \pm 4.02$ & $26.66 \pm 2.94$ & 1.19 & 1.09 & $1664 \pm 190$ \\
$\mathbf{6}$ & $20.85 \pm 1.32$ & $18.73 \pm 1.02$ & 0.90 & 3.40 & $1347 \pm 110$ \\
$\mathbf{7}$ & $27.34 \pm 2.35$ & $26.75 \pm 2.75$ & 0.98 & 2.08 & $1549 \pm 82$ \\
$\mathbf{8}$ & $26.21 \pm 3.54$ & $23.34 \pm 1.67$ & 0.89 & 2.17 & $1298 \pm 161$ \\
$\mathbf{9}$ & $43.62 \pm 2.13$ & $37.38 \pm 5.81$ & 0.86 & 2.17 & $1595 \pm 173$ \\
$\mathbf{1 0}$ & $7.29 \pm 0.17$ & $3.73 \pm 0.33$ & 0.51 & 3.7 & $1615 \pm 136$ \\
$\mathbf{1 1}$ & $10.42 \pm 0.95$ & $7.12 \pm 0.12$ & 0.68 & 4.28 & $1429 \pm 152$ \\
$\mathbf{1 2}$ & $38.34 \pm 1.72$ & $33.74 \pm 6.35$ & 0.88 & 2.54 & $1463 \pm 119$ \\
\hline caffeine $(10 \mu \mathrm{M})$ & $43.58 \pm 4.86$ & & & & $1635 \pm 53$ \\
Atenolol $(200 \mu \mathrm{M})$ & $0.76 \pm 0.16$ & & & & $1573 \pm 91$ \\
\hline
\end{tabular}

$P_{a p p \text { AP-BL: }}$ transport from AP to BL side; $P_{a p p \text { BL-AP: }}$ transport from BL to AP side; $P_{a p p \text { BL-AP }} / P_{a p p \text { AP-BL}}:$ efflux ratio of $P_{a p p}$ BL-AP to $P_{a p p}$ AP-BL. The concentration of all coumarins was $50 \mu \mathrm{M}$. The incubation time was up to 90 min. Data are means \pm S.D. $(n=6)$.

Rhodamine 123 (Rh123), a typical probe substrate for P-gp, has been widely used in P-gp researches. When co-incubated with the P-gp inhibitor verapamil, Rh123 efflux was inhibited with the

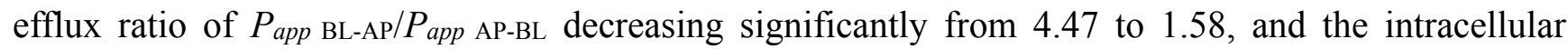
accumulation of Rh123 was 4.45-fold higher than that of verapamil-absent group (Table 2). All results indicated that P-gp was stably expressed in the MDCK-pHaMDR cell and played obvious efflux effect on drug transport.

Table 2. Rh123 transportation and intracellular accumulation in MDCK-pHaMDR cell.

\begin{tabular}{|c|c|c|c|c|}
\hline \multirow{2}{*}{ Compound } & \multicolumn{2}{|c|}{$P_{a p p}\left(\times 10^{-7} \mathrm{~cm} / \mathrm{s}\right)$} & \multirow{2}{*}{$\boldsymbol{P}_{a p p \text { BL-AP }} / \boldsymbol{P}_{a p p} \mathrm{AP}-\mathrm{BL}$} & \multirow{2}{*}{$\begin{array}{l}\text { Intracellular Accumulation } \\
\text { Fluorescence Intensity }\end{array}$} \\
\hline & AP-BL & BL-AP & & \\
\hline Rh123 & $11.81 \pm 0.56$ & $52.74 \pm 2.09$ & $4.47 \pm 0.24$ & $34.51 \pm 1.18$ \\
\hline Rh123 + $100 \mu \mathrm{M}$ verapamil & $5.36 \pm 0.41$ & $8.51 \pm 1.43$ & $1.58 \pm 0.208 *$ & $153.64 \pm 3.28 *$ \\
\hline
\end{tabular}

\subsection{Bidirectional Transport Determination}

The permeation samples were examined by HPLC since coumarins had good ultraviolet absorptions and the maximum wavelength was chosen for each compound. Rapid, simple and reliable HPLC methods had been established for the analysis of twelve coumarins. The standard calibration curves were constructed by plotting peak area $(\mathrm{y}) v s$. concentration $(\mathrm{x}, \mu \mathrm{M})$. Regression equation, coefficient correlation (r) of the methods for twelve coumarins were shown as follow: $y=14.82 x+4.21(0.9998$, 
for 1), $y=10.47 x-021.85(0.9992$, for 2$), y=12.26 x-10.59(0.9997$, for 3$), y=11.95 x+8.62(0.9993$, for 4), $y=18.44 x+3.58(0.9998$, for 5$), y=16.32 x-14.86(0.9998$, for 6), $y=23.50 x-28.19(0.9995$, for 7), $y=12.59 x+0.44(0.9997$, for 8), $y=10.76 x+9.68(0.9995$, for 9), $y=7.46 x+3.70(0.9998$, for 10), $y=12.58 \mathrm{x}-22.87$ (0.9993, for 11), $\mathrm{y}=18.25 \mathrm{x}-6.53$ (0.9997, for 12), respectively. The concentration range of the calibration curve was $0.5-150 \mu \mathrm{M}$ for $\mathbf{4}, \mathbf{5}, \mathbf{7}, \mathbf{8}, 1-150 \mu \mathrm{M}$ for $\mathbf{1}, \mathbf{2}, \mathbf{3}, \mathbf{6}, \mathbf{9}, \mathbf{1 0}$, 12, and $2-150 \mu \mathrm{M}$ for $\mathbf{1 1}$, respectively. The intraday and interday precision of the methods for twelve test coumarins were less than $3.41 \%$ and $3.79 \%$, respectively, and the accuracies of the methods were between $85.18 \%$ and $107.71 \%$. The concentrations after three freeze-thaw cycles changed from $95.71 \%$ to $103.97 \%$, or less than $\pm 5 \%$ range. The data, summarized in Supplemental Materials Table S1, proves the HPLC methods were feasible.

In general, drugs with high $P_{a p p}\left(>1 \times 10^{-5} \mathrm{~cm} / \mathrm{s}\right)$ can be well-absorbed, while those with low $P_{a p p}$ $\left(<1 \times 10^{-6} \mathrm{~cm} / \mathrm{s}\right)$ are poorly absorbed [23]. The bidirectional $P_{\text {app }}$ values for coumarins $\mathbf{1}-\mathbf{1 2}$ have been

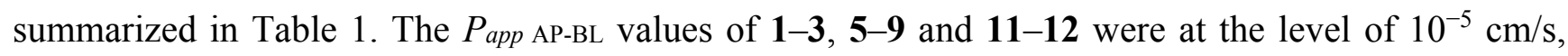
similar to that of caffeine, so they were classified as well-absorbed compounds through the BBB. Coumarins $\mathbf{4}$ and $\mathbf{1 0}$ were assigned to the moderately absorbed compounds, since their $P_{a p p}$ AP-BL values of $10^{-6} \mathrm{~cm} / \mathrm{s}$ laid between the levels of caffeine and atenolol. The possible pathway of most test coumarins was passive transport because the ratios of $P_{a p p}$ BL-AP/ $/ P_{a p p}$ AP-BL were less than 2.0 [23].

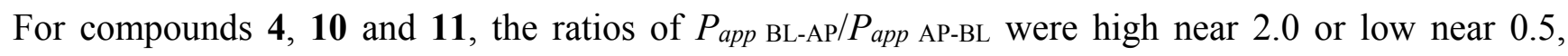
so P-gp inhibitor verapamil was further used to verify their absorption pathway.

To check the intracellular accumulation and mass balance, the recoveries of twelve coumarins (total amounts of the compounds in both sides of the insert and intracellular) were measured. The test coumarins had high recoveries of $>85 \%$ and low intracellular accumulations of $<7 \%$, except compound 11 (Supplemental Materials Table S2). As for compound 11, the relative low recovery $(64 \%-68 \%)$ and high intracellular accumulation (9\%-22\%) were clearly different from those of the other coumarins.

\subsection{Time- and Concentration-Dependent Permeation}

The bidirectional time-permeation curves of twelve test coumarins at $50 \mu \mathrm{M}$ are presented in Figures 2 and 3. Concentration difference between membrane sides is one of the important features of passive diffusion. When the concentration difference of test compounds between the receiver side and donor side reaches a certain degree, the transport rate will slow down. Both AP-BL and BL-AP transport cumulative amounts of all compounds at $50 \mu \mathrm{M}$ increased almost linearly with time, while the transport rates of all compounds except $\mathbf{1 0}$ decreased linearly with time, according to the transport balance of concentration saturation. The slight increase transport rate of $\mathbf{1 0}$ in 60 min maybe relate with a long time to reach concentration saturation because of its smallest bidirectional $P_{a p p}$. The bidirectional transport rates of most test coumarins increased linearly within the concentration range of 10-125 $\mu \mathrm{M}$ (Figure 4), except that the transport rates of $\mathbf{4}$ and $\mathbf{8}$ in AP-BL direction and 6, 8, 11 in BL-AP direction had slower increase in high concentrations (from $75 \mu \mathrm{M}$ ). For these compounds, since the linear trends were obvious in the concentrations under $75 \mu \mathrm{M}$ but close to slow platform from 75 to $125 \mu \mathrm{M}$, a possible explanation was that the drug saturation on the receiver side slowed down the transport at higher concentrations. 

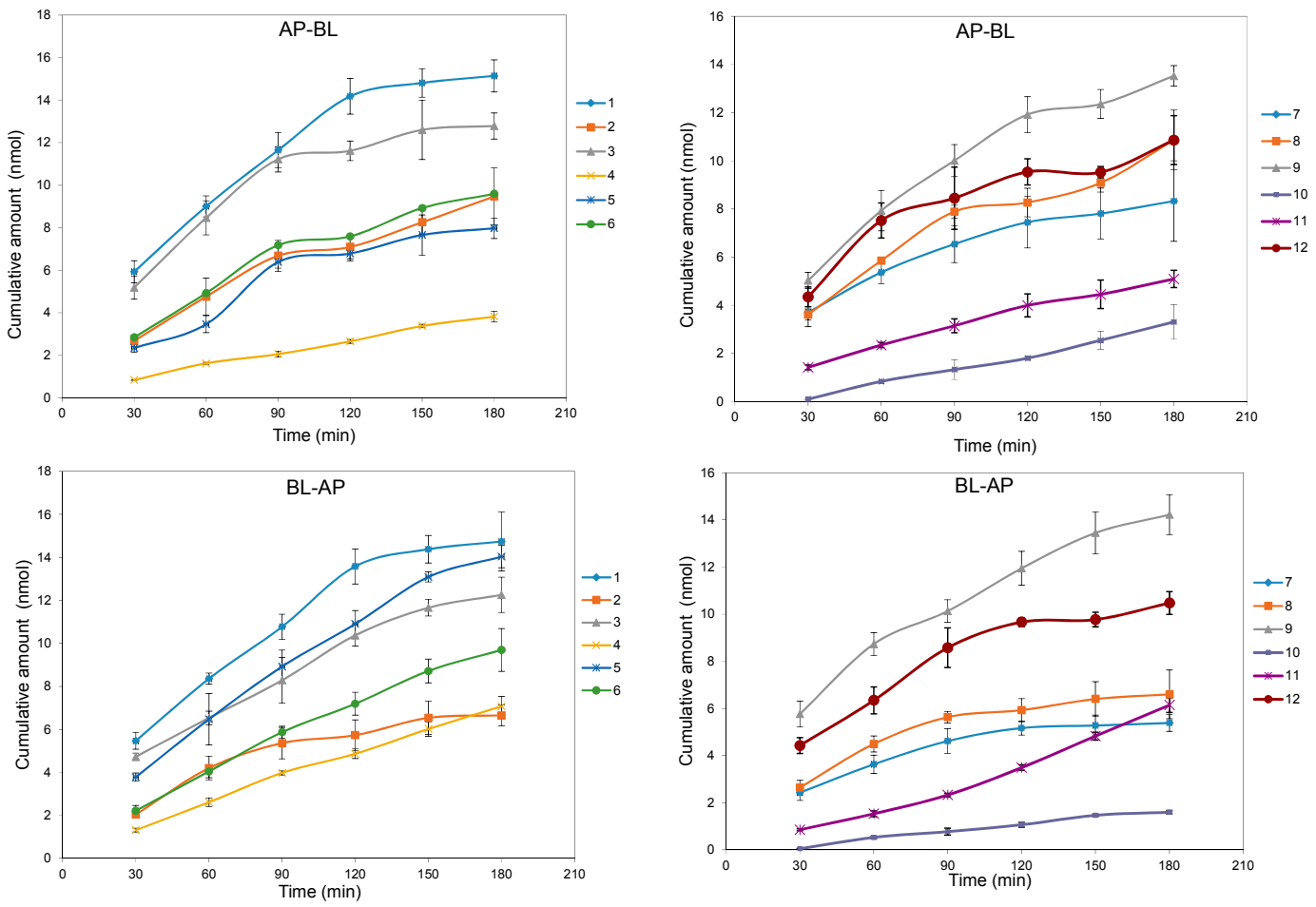

Figure 2. The bidirectional transport cumulative amounts of twelve coumarins in MDCK-pHaMDR cell monolayer as a function of time at $50 \mu \mathrm{M}$. Data are the mean \pm S.D. $(n=6)$.
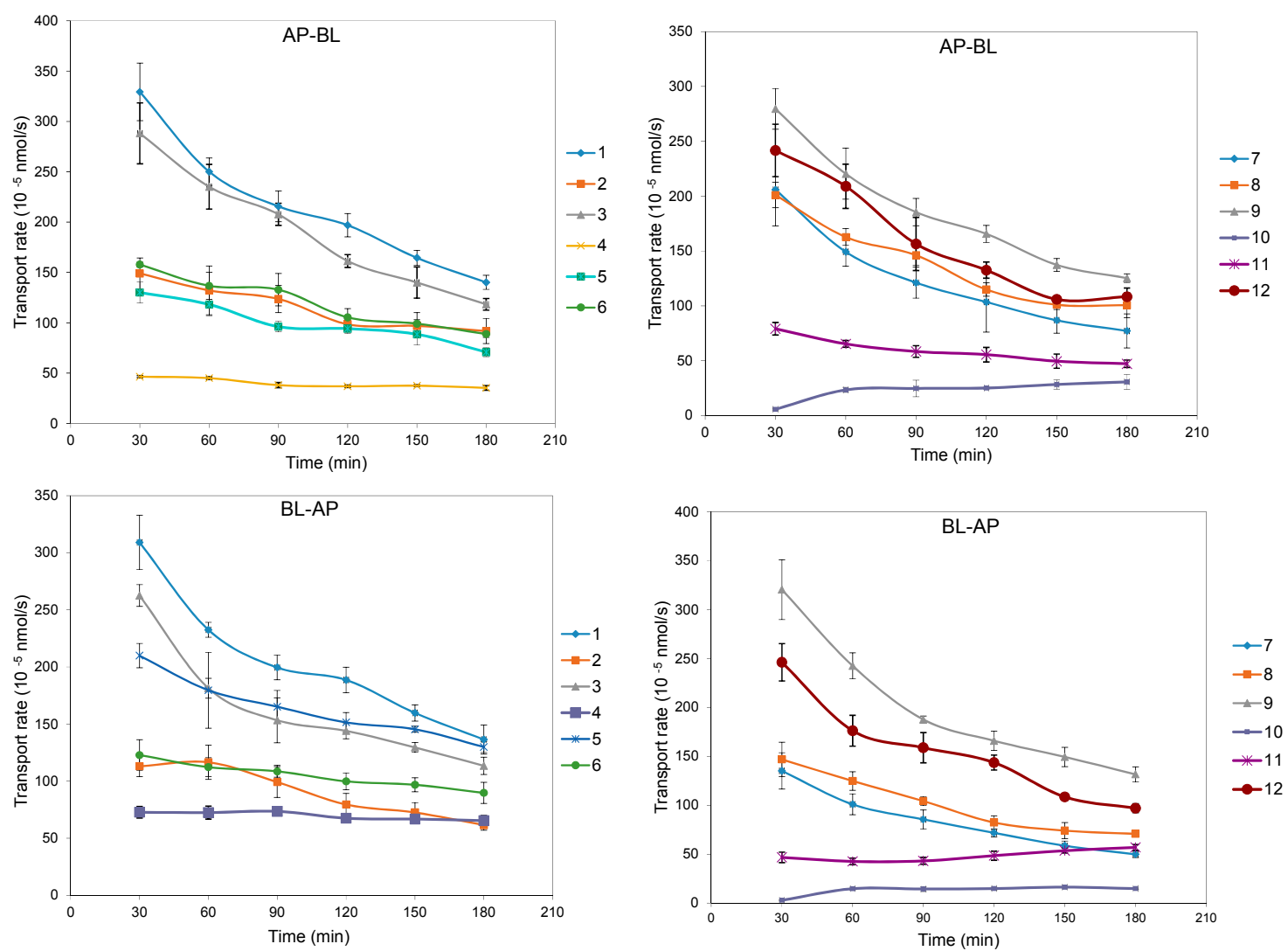

Figure 3. The bidirectional transport rate of twelve coumarins in MDCK-pHaMDR cell monolayer as a function of time at $50 \mu \mathrm{M}$. Data are the mean \pm S.D. $(n=6)$. 

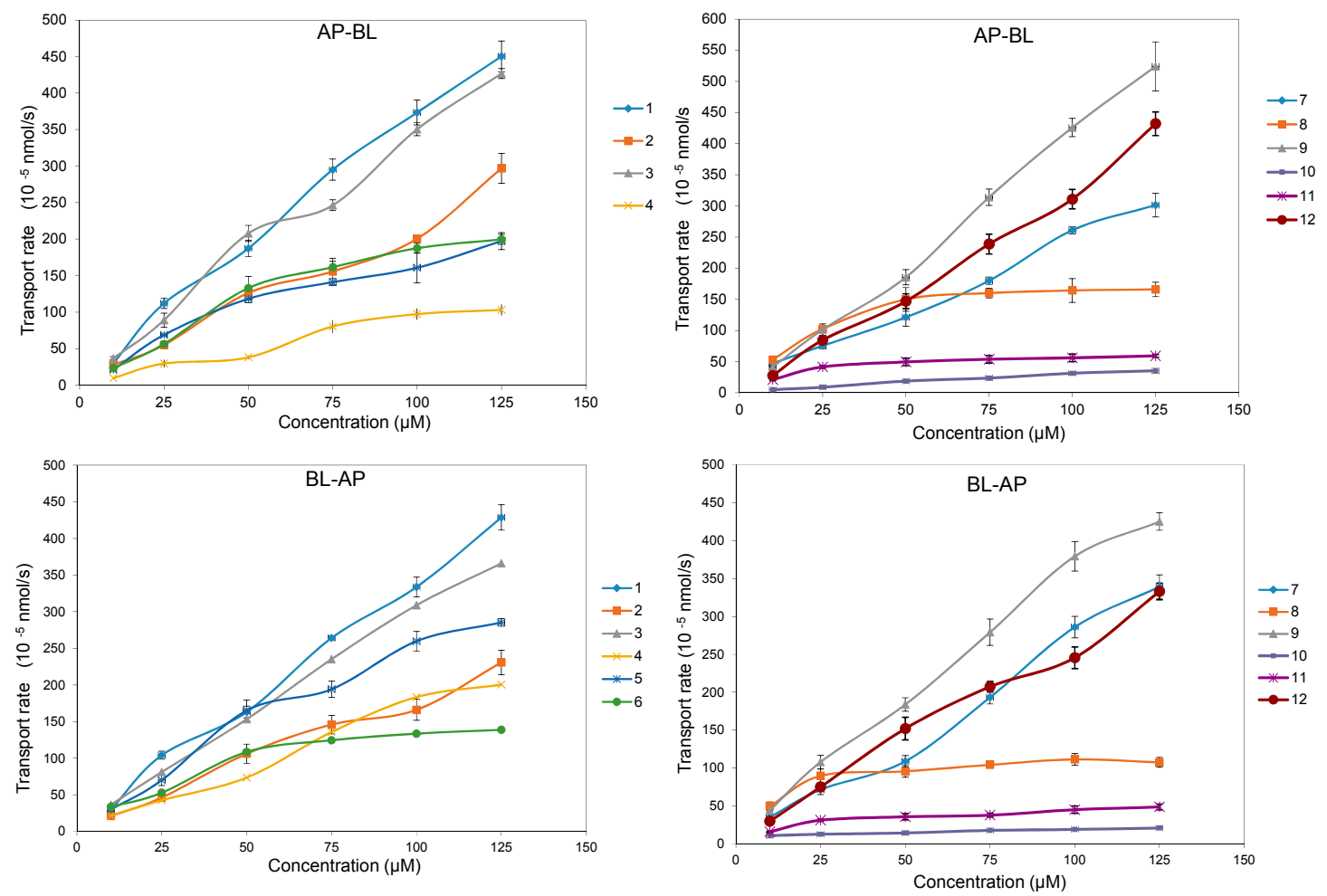

Figure 4. The bidirectional transport rate of twelve coumarins in MDCK-pHaMDR cell monolayer as a function of concentration at $10-125 \mu \mathrm{M}$. Data are the mean \pm S.D. $(n=6)$.

\subsection{Transport Inhibition by Verapamil}

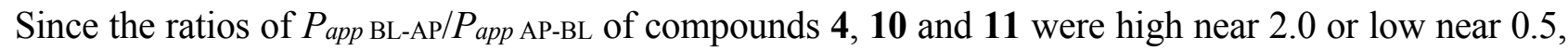
verapamil at $100 \mu \mathrm{M}$ was used as a selective P-gp inhibitor during the transport experiment. When bidirectional transports of selected coumarins toghter with $100 \mu \mathrm{M}$ verapamil were studied, the ratio of

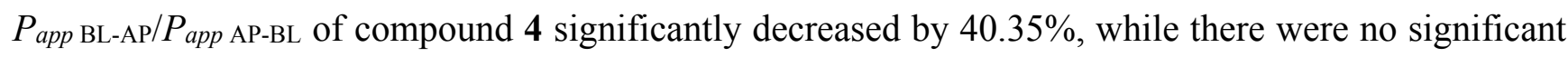
changes of compounds $\mathbf{1 0}$ and $\mathbf{1 1}$ (Figure 5). The results revealed that P-gp related efflux mechanism was involved in the transport of compound 4, while P-gp had no effect on the absorption process of coumarins $\mathbf{1 0}$ and $\mathbf{1 1 .}$

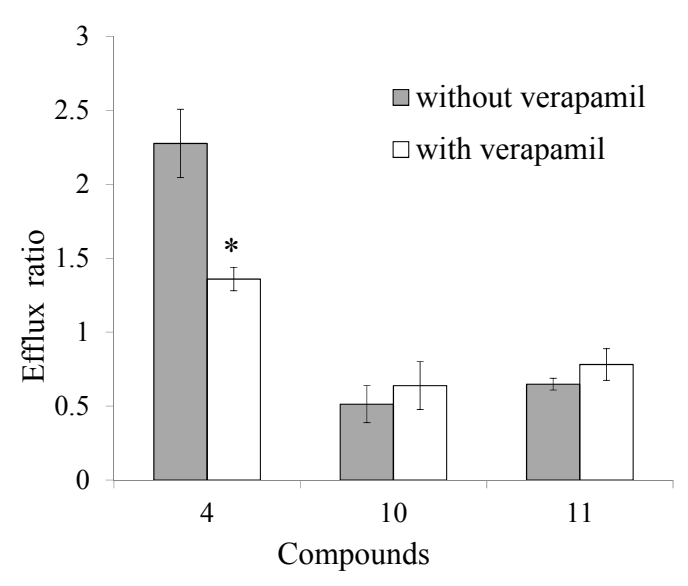

Figure 5. The efflux ratios of coumarins 4, 10, 11 in MDCK-pHaMDR cell in the absence or presence of $100 \mu \mathrm{M}$ verapamil. ${ }^{*} p<0.01 v s$. verapamil absent group. 


\subsection{Structure-Permeability Relationship Analysis}

To analyze the structure-permeability relationship, $P_{a p p}$ values of the test coumarins were compared. There were significant differences in $P_{a p p}$ values between $\mathbf{1}$ and 2, as well as $\mathbf{7}$ and $\mathbf{1 0}$ when contrasted, which indicated that the straight chain substitutions on the coumarin nucleus may decrease the coumarin penetration through the BBB. The $P_{a p p}$ value similarities between $\mathbf{1}$ and $\mathbf{3}$, as well as $\mathbf{7}$ and $\mathbf{8}$, showed that the methoxyl group substitutent on the aromatic ring had no effect on the coumarin penetration through the BBB, but the different substituent positions of the methoxyl group may have obvious effects on the coumarin penetrations according to the $P_{\text {app }}$ comparison between 8 and $\mathbf{9}$. The $P_{\text {app }}$ difference between $\mathbf{4}$ and 5 revealed that methoxylation of a hydroxyl group can obviously promote the penetration of coumarins. The comparison between $\mathbf{1 1}$ and $\mathbf{1 2}$ indicated that an angeloyl substituent maybe give lower $P_{a p p}$ values than an acetyl group. In addition, the $P_{a p p}$ difference between $\mathbf{1}$ and $\mathbf{7}$ showed that a furan ring substituted at $\mathrm{C}_{6}$ and $\mathrm{C}_{7}$ of the aromatic ring may decrease the coumarin absorption across the $\mathrm{BBB}$.

Physicochemical characters, such as the logarithm of distribution-coefficient (log D), the logarithm of partition-coefficient $(\log \mathrm{P})$, and polar surface area, are generally utilized for the prediction of drug permeability. In this study, it was notable that the coumarin penetrations across MDCK-pHaMDR cell monolayer were not well correlated with their $\log$ P. Theoretical passive drug absorption [28] and experimental transport across intestinal epithelial cells [29] have proved that lipophilicity has a close relationship with the tansepithelial permeability of compounds. Our previous works have demonstrated the sigmoid relationship between permeability $\log \left(P_{\text {app AP-BL }} * \mathrm{MW}^{0.5}\right)$ and $\log \mathrm{D}$ of neolignans [30] and coumarins [20] in Caco-2 cell monolayer. In this study, $\log \mathrm{D}$ (at pH 7.4) was chosen as the index of lipophilicity, calculated with Pallas 3.3.2.6 ADME/Tox Software (CompuDrug, Bal Harbor, FL,USA) (Table 1). Two sigmoid functions linked at $\log \mathrm{D} 2.5$ were resulted by plotting $\log \left(P_{\text {app }} \mathrm{AP}-\mathrm{BL} * \mathrm{MW}^{0.5}\right) v s$. $\log$ D (at pH 7.4) with Matlab (R2013a, MathWorks, Natick, MA, USA) (Figure 6) and the equations are listed as follow:

$$
f(x)= \begin{cases}\frac{1}{1+\mathrm{e}^{-2.9 x-2.25}}-4.227 & \mathrm{x} \leq 2.5 \\ \frac{1}{1+\mathrm{e}^{3.3 \mathrm{x}-13.25}}-4.227 & \mathrm{x}>2.5\end{cases}
$$

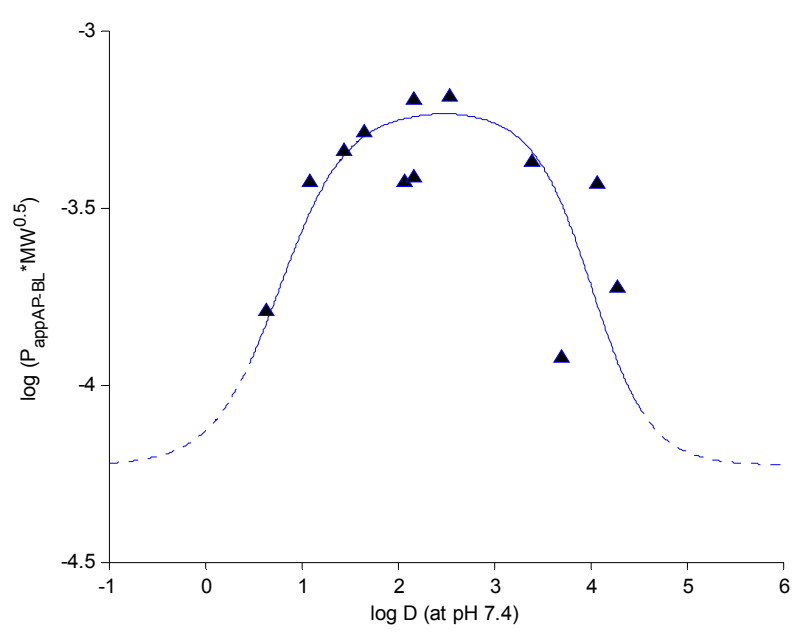

Figure 6. The relationship between permeability $\log \left(P_{\text {app }} \mathrm{AP}-\mathrm{BL} * \mathrm{MW}^{0.5}\right)$ and $\log \mathrm{D}$ (at $\mathrm{pH}$ 7.4) of twelve coumarins. 
The sigmoid curve suggested that the BBB transepithelial permeabilities of coumarins (compounds $\mathbf{1}$, $3,5,6,7,8,9,12)$ with moderate $\log \mathrm{D}(1.0-3.5)$ were relatively high and less affected by the change of $\log \mathrm{D}$, while the penetrations of those with higher $\log \mathrm{D}(3.5-4.5)(\mathbf{2}, \mathbf{1 0}, \mathbf{1 1})$ or $\operatorname{lower} \log \mathrm{D}(<1.0)(\mathbf{4})$ decreased rapidly when $\log \mathrm{D}$ changed. The result indicated that too high or too low lipophilicity may result in low BBB permeability, which agreed with the researches of intestinal permeability [29]. Therefore $\log \mathrm{D}$ as the index of lipophilicity was important and useful for predicting drug BBB permeability.

\section{Experimental Section}

\subsection{Chemicals and Reagents}

Twelve coumarins, umbelliferone (1), osthol (2), scopoletin (3), peucedanol (4), ulopterol (5), angepubebisin (6), psoralen (7), xanthotoxin (8), bergapten (9), isoimperatorin (10), columbianadin (11), columbianetin acetate (12), were isolated from APR and characterized in our group [31,32]. The purities were determined to be above $98.0 \%$. Colchicine, caffeine, atenolol, dimethylsulfoxide (DMSO) were purchased from Sigma-Aldrich (St. Louis, MO, USA). Methanol (MeOH) was of HPLC grade (J. T. Backer, Center Valley, PA, USA). Milli-Q water (Millipore, Bedford, MA, USA) was used throughout the study. Other chemicals were of analytical grade.

\subsection{Cell Culture}

MDCK-pHaMDR cell line was a gift from Dr. Michael M. Gottesman (NIH, Bethesda, MD, USA). Dulbecco's Mocified Eagle's Medium (DMEM), Fetal bovine serum (FBS), trypsin, penicillin, streptomycin and other culture media and supplements were obtained from Gibco (Life Science Technologies, Carlsbad, CA, USA). Reagents for Hank's Balanced Salts Solution (HBSS) were from Beijing Chemical Works (Beijing, China). 3-(4,5-Dimethyl-2-thiazolyl)-2,5-diphenyl-2H-tetrazolium bromide (MTT), Rh123 and verapamil were purchased from Sigma-Aldrich (St. Louis, MO, USA). 12 Wells Transwell ${ }^{\circledR}$ plates with polycarbonate inserts $(3 \mu \mathrm{m}$ pore size and $12 \mathrm{~mm}$ in diameter $)$ and 6 Wells plates were obtained from Corning Costar (Cambridge, MA, USA). The cytotoxicity of the test compounds on the MDCK-pHaMDR cells was determined with the MTT assay on a Thermo Multiskan MK3 Automated Microplate Reader (Thermo-Labsystems, Franklin, MA, USA).

The cells were cultured at $37{ }^{\circ} \mathrm{C}$ in $5 \% \mathrm{CO}_{2}$, using DMEM with $4.5 \mathrm{~g}$ of glucose per liter and $1 \mathrm{mM}$ sodium pyruvate supplemented with $10 \% \mathrm{FBS}, 5 \mathrm{mM}$ L-glutamine, 50 units $/ \mathrm{mL}$ penicillin and $50 \mu \mathrm{g} / \mathrm{mL}$ streptomycin. Cells were harvested with $0.25 \%$ trypsin-EDTA and seeded onto the Transwell insert filter at a density of about $8 \times 10^{4}$ cells $/ \mathrm{mL}$. After growing for 8 days, the cells reached confluence and full differentiation for the transport experiments [26]. All cells used in this study were between passages 8 and 30 .

\subsection{Transport Experiments}

$10 \mathrm{mM}$ stock solutions of test compounds were prepared in DMSO and diluted with HBSS to the desired concentrations before transport experiments. HBSS ( $\mathrm{pH} 7.4$ ) was used as a transport medium. After washing the MDCK-pHaMDR cell monolayer twice with prewarmed HBSS medium, HBSS medium was preserved to prepare samples of the calibration curves. Then the experiments were carried 
out by replacing the transport medium in the AP side $(0.5 \mathrm{~mL}$, for absorption transport $)$ or $\mathrm{BL}$ side (1.5 mL, for efflux transport) with test compounds $(50 \mu \mathrm{M})$. The plates were shaked at $55 \mathrm{rpm}$ for $90 \mathrm{~min}$ at $37{ }^{\circ} \mathrm{C}$ in a water bath. Samples were collected from both sides of the cell monolayer, then immediately frozen, lyophilized and preserved below $-20^{\circ} \mathrm{C}$. To measure the intracellular accumulation amounts of different coumarins, the cell monolayers were extracted after transport assays with $200 \mu \mathrm{L}$ $70 \% \mathrm{MeOH}(v / v)$ for $20 \mathrm{~min}$, then centrifuged and preserved below $-20{ }^{\circ} \mathrm{C}$.

\subsection{Standard Conditions of MDCK-pHaMDR Cell Monolayer}

The integrity and transport capacity of the MDCK-pHaMDR cell monolayer were examined by measuring the TEER with an epithelial voltohmmeter (EVOM, World Precision Instrument, Sarasota, FL, USA). Only cell monolayer with a TEER above $1000 \Omega \cdot \mathrm{cm}^{2}$ was used for the transport assay [26]. Standard compounds, caffeine and atenolol were run as the active and passive transport marker, respectively.

The P-gp expression level in the MDCK-pHaMDR cell monolayer was examined by Rh123 transport and intracellular accumulation experiments. Rh123 $(10 \mu \mathrm{M})$ bidirectional transports with or without the presence of verapamil $(100 \mu \mathrm{M})$ were undertaken as described above, and the lyophilized permeation samples were dissolved in a proper volume of $\mathrm{MeOH}$. The fluorescence at $485 \mathrm{~nm}$ (excitation wavelength) and $538 \mathrm{~nm}$ (emission wavelength) was determined on a Cary Eclipse Fluorescence spectrophotometer (Varian Inc., Palo Alto, CA, USA). To investigate intracellular accumulations of Rh123 as a probe substrate for P-gp, MDCK-pHaMDR cells were seeded on the 6 Wells plates at a density of about $1.0 \times 10^{5}$ cells $/ \mathrm{mL}$ and grew for $48 \mathrm{~h}$. Before the uptake experiments, cells were washed with PBS two times and pre-incubated with serum-free DMEM or $100 \mu \mathrm{M}$ verapamil for $20 \mathrm{~min}$. Rh123 with the final concentration of $10 \mu \mathrm{M}$ was added, and cells were incubated with or without the presence of verapamil for $60 \mathrm{~min}$ at $37^{\circ} \mathrm{C}$. Then cells were washed with ice-cold PBS three times, and lysed with $0.1 \%(v / v)$ Triton X-100 for $15 \mathrm{~min}$ at $37^{\circ} \mathrm{C}$ [33]. The fluorescence of Rh123 in cell lysates was determined as described above.

\subsection{Time- and Concentration-Dependent Transport Experiments}

The permeable coumarins were chosen to study the time- and concentration-dependence permeability across the MDCK-pHaMDR cell monolayer. To observe the time-dependence of the selected coumarin, test compounds at $50 \mu \mathrm{M}$ were added to either AP or BL side of the inserts for absorption transport (AP-BL) or efflux transport (BL-AP), and incubated for 30, 60, 90, 120, 150 and $180 \mathrm{~min}$. In the concentration-dependent transport study, 10, 25, 50, 75, 100, and $125 \mu \mathrm{M}$ of certain coumarins were added to either AP or BL side and incubated for 90min. Transport experiments were undertaken as described above.

\subsection{Verapamil Inhibition of Transport}

The verapamil inhibition experiments were carried out for compounds $4, \mathbf{1 0}$ and $\mathbf{1 1}$. The cells were pre-incubated with $100 \mu \mathrm{M}$ verapamil for $30 \mathrm{~min}$ before drug transport assays. Then the inhibitor was added to both sides of the membrane and the coumarins $(4,10$ and 11$)$ at $50 \mu \mathrm{M}$ were added to AP or 
BL side. Coumarin transport experiments were undertaken as described above and the efflux ratios of Papp BL-AP/Papp AP-BL were calculated.

\subsection{HPLC Analysis}

An Agilent 1100 series HPLC system (Agilent Technologies, Palo Alto, CA, USA) was used with an analytical Diamonsil ${ }^{\circledR} \mathrm{C}_{18}$ column $(250 \mathrm{~mm} \times 4.6 \mathrm{~mm}, 5 \mu \mathrm{m}$, Dikma, Beijing, China) equipped with a $\mathrm{C}_{18}$ guard column ( $8 \mathrm{~mm} \times 4 \mathrm{~mm}, 5 \mu \mathrm{m}$, Dikma). The mobile phase was composed of $\mathrm{MeOH}-\mathrm{H}_{2} \mathrm{O}$ $(v / v)$ in $68: 32$ for $\mathbf{1}, 90: 10$ for $\mathbf{2}, 53: 47$ for $\mathbf{3}, 55: 45$ for $\mathbf{4}, 66: 34$ for $\mathbf{5}, 88: 12$ for $\mathbf{6}, 75: 25$ for $\mathbf{7}, 80: 20$ for $\mathbf{8}, 70: 30$ for $\mathbf{9}, 92: 8$ for $\mathbf{1 0}, \mathbf{7 5 : 2 5}$ for $\mathbf{1 1}, \mathbf{7 8 : 2 2}$ for $\mathbf{1 2}$, and the UV detection was at $320 \mathrm{~nm}$ for $\mathbf{1}$, $330 \mathrm{~nm}$ for $\mathbf{2}, \mathbf{4}, \mathbf{5}, 11$ and $\mathbf{1 2}, 344 \mathrm{~nm}$ for $\mathbf{3}, 325 \mathrm{~nm}$ for $\mathbf{6}, 250 \mathrm{~nm}$ for 7, $310 \mathrm{~nm}$ for $\mathbf{8}$ and $\mathbf{1 0}, 300 \mathrm{~nm}$ for 9 . The flow rate was $1.0 \mathrm{~mL} / \mathrm{min}$. Elution peaks were monitored and the peak areas were used to calculate the compound concentrations.

To evaluate linearity, calibration curves were prepared and assayed. Accuracy and precision were assessed by determining quality control (QC) samples at three concentration levels $(5,50$ and $150 \mu \mathrm{M})$ on three different consecutive days. The recoveries at three QC levels were also determined. The freeze-thaw stabilities were assessed by analyzing the QC samples undergoing three freeze $\left(-20^{\circ} \mathrm{C}\right.$ )-thaw (room temperature) cycles. To determine the corresponding coumarins, the lyophilized permeation samples were dissolved in a suitable volume of $\mathrm{MeOH}$, thoroughly vortex-mixed for $1 \mathrm{~min}$ and then centrifuged at $16,000 \times g$ for $20 \mathrm{~min}$. An aliquot of $20 \mu \mathrm{L}$ supernatant solution was used for HPLC assay. To measure the intracellular accumulation amount of different coumarins, the aliquots of cell extractions were determined too.

\subsection{Data Analysis}

$P_{a p p}$ in AP-BL or BL-AP direction of each coumarin was calculated from the following equation:

$$
P_{a p p}=\frac{\mathrm{dQ}}{\mathrm{dt} \cdot \mathrm{A} \cdot \mathrm{C}_{0}}[\mathrm{~cm} / \mathrm{s}]
$$

where $\mathrm{Q}$ is the accumulation quantity of the compound in the receiver side $(\mu \mathrm{mol}), \mathrm{dQ} / \mathrm{dt}$ is the linear appearance rate of the compound in the receiver side $(\mu \mathrm{mol} / \mathrm{s}), \mathrm{C}_{0}$ is the initial concentration in the donor side $(\mu \mathrm{M})$, and $\mathrm{A}$ is the surface area of the membrane insert $\left(\mathrm{cm}^{2}\right)$. Data were expressed as the mean $\pm \mathrm{SD}$.

\section{Conclusions}

In conclusion, most test coumarins can be transported across the MDCK-pHaMDR cell monolayer by passive diffusion and are well or moderately absorbed. The compound structure may have an effect on the coumarin penetration capacity, and $\log \mathrm{D}$ values may have important relationship with the permeability through the BBB. The present results provide some useful information for studying the CNS activity of APR and predicting the penetration capacity of coumarins through the BBB. Based on the results, our next study objective will be to observe the brain distributions of the main coumarins of APR and carry out a comparison between the in vivo and in vitro absorption characteristics of coumarins. In addition, according to the polarized expression of P-gp in the MDCK-pHaMDR cell line and our previous works on $\log \mathrm{D}$ related permeability studies, the results could be explained on the basis of a P-gp efflux-related 
transport mechanism and lipophilicity-permeability relationship analysis. Since there are other enzymes, proteins and receptors to cover the whole functional features of the BBB, there are some limitations of the findings and further experiments about other factors of BBB are expected.

\section{Supplementary Materials}

HPLC method validations, together with the cell accumulation and total recovery of coumarin transport are available as Supplementary Materials, which may be accessed at: http://www.mdpi.com/ 1420-3049/20/07/11719/s1.

\section{Acknowledgments}

The authors thank Michael M. Gottesman at NIH supplies the MDCK-pHaMDR cell line for this study. The work was supported by the National Nature Science Foundation of China (No. 81403068) and the National Key Technology R \& D Program of China (No. 2011BAI07B08).

\section{Author Contributions}

The list authors contributed to this work as follows: X.-W. Y. conceived and designed the experiments, Y.-F. Y., W. S. and W. X. performed the experiments and analyzed the data, Y.-F. Y. wrote the paper, X.-W. Y. and M. Y. polished the paper. X.-W. Y. and Y.-F. Y. acquired funding for the research. All authors read and approved the final manuscript.

\section{Conflicts of Interest}

The authors declare no conflict of interest.

\section{References}

1. Chen, Y.F.; Tsai, H.Y.; Wu, T.S. Anti-inflammatory and analgesic activities from roots of Angelica pubescens. Planta Med. 1995, 61, 2-8.

2. Li, X.; Wang, J.; Gao, L. Anti-inflammatory and analgesic activity of R.A.P. (Radix Angelicae Pubescentis) ethanol extracts. Afr. J. Tradit. Complement. Altern. Med. 2013, 10, 422-426.

3. Sun, J.; Liu, B.R.; Hu, W.J.; Yu, L.X.; Qian, X.P. In vitro anticancer activity of aqueous extracts and ethanol extracts of fifteen traditional Chinese medicines on human digestive tumor cell lines. Phytother. Res. 2007, 21, 1102-1104.

4. Liu, J.H.; Xu, S.X.; Yao, X.S.; Kobayashi, H. Angelol-type coumarins from Angelica pubescens f. biserrata, and their inhibitory effect on platelet aggregation. Phytochemistry 1995, 39, 1099-1101.

5. Liao, J.F.; Jan, Y.M.; Huang, S.Y.; Wang, H.H.; Yu, L.L.; Chen, C.F. Evaluation with receptor binding assay on the water extracts of ten CNS-active Chinese herbal drugs. Proc. Natl. Sci. Counc. Repub. China B 1995, 19, 151-158.

6. Pei, Y.; Li, D.X.; Sun, S.H. Experimental study on the effect of Radix Angelicae Pubescentis and its alcohol extract on apoptosis of brain cells in nature aging mice. Chin. J. Gerontol. 2005, 25, 959.

7. Hu, Y.; Zhao, D.; Zhang, X.D.; Sun, D.; Hao, H.G.; Yang, J.X. Different extracts of Angelica pubescens inhibit $\mathrm{H}_{2} \mathrm{O}_{2}$-induced SH-SY5Y cells injury. Chin. J. Exp. Tradit. Med. Form. 2013, 19, 184-188. 
8. Zhu, M.D.; Cui, J.P.; Wang, C.X. The experimental study of the intervention effect of Duhuo on immune damage of model rats suffering from Alzheimer. Liaoning J. Tradit. Chin. Med. 2011, 38, 2085-2086.

9. Liu, J.H.; Xu, S.X.; Yao, X.S. Chemical Constituents and Pharmacology Research Review of Angelicae Pubescentis Radix. J. Shenyang Coll. Pharm. 1994, 11, 143-150.

10. Hornick, A.; Lieb, A.; Vo, N.P.; Rollinger, J.M.; Stuppner, H.; Prast, H. The coumarin scopoletin potentiates acetylcholine release from synaptosomes, amplifies hippocampal long-term potentiation and ameliorates anticholinergic- and age-impaired memory. Neuroscience 2011, 197, 280-292.

11. Witaicenis, A.; Seito L.N.; da Silveira Chagas, A.; de Almeida, L.D.; Luchini, A.C.; Rodrigues-Orsi, P.; Cestari, S.H.; Di Stasi, L.C. Antioxidant and intestinal anti-inflammatory effects of plant-derived coumarin derivatives. Phytomedicine 2014, 21, 240-246.

12. Hu, Y.; Wen, Q.; Liang, W.; Kang, T.; Ren, L.; Zhang, N.; Zhao, D.; Sun, D.; Yang, J. Osthole reverses beta-amyloid peptide cytotoxicity on neural cells by enhancing cyclic AMP response element-binding protein phosphorylation. Biol. Pharm. Bull. 2013, 36, 1950-1958.

13. He, Y.; Qu, S.; Wang, J.; He, X.; Lin, W.; Zhen, H.; Zhang, X. Neuroprotective effects of osthole pretreatment against traumatic brain injury in rats. Brain Res. 2012, 1433, 127-136.

14. Kim, D.K.; Lim, J.P.; Yang, J.H.; Eom, D.O.; Eun, J.S.; Leem, K.H. Acetylcholinesterase inhibitors from the roots of Angelica dahurica. Arch. Pharm. Res. 2002, 25, 856-859.

15. Kong, L.D.; Tan, R.X.; Woo, A.Y.; Cheng, C.H. Inhibition of rat brain monoamine oxidase activities by psoralen and isopsoralen: Implications for the treatment of affective disorders. Pharmacol. Toxicol. 2001, 88, 75-80.

16. Huong, D.T.; Choi, H.C.; Rho, T.C.; Lee, H.S.; Lee, M.K.; Kim, Y.H. Inhibitory activity of monoamine oxidase by coumarins from Peucedanum japonicum. Arch. Pharm. Res. 1999, 22, 324-326.

17. Sigurdsson, S.; Gudbjarnason, S. Inhibition of acetylcholinesterase by extracts and constituents from Angelica archangelica and Geranium sylvaticum. Z. Naturforsch. C 2007, 62, 689-693.

18. Yang, X.W.; Guo, Q.M.; Wang, Y. Absorption and transport of six coumarins isolated from the roots of Angelica pubescens f. biserrata in human Caco-2 cell monolayer model. J. Chin. Integr. Med. 2008, 6, 392-398.

19. Long, J.; Li, S.N.; Yang, X.W. Absorption characteristics of four linear furocoumarins across human intestinal epithelial in a model of Caco-2 cell monolayer. Chin. J. New Drugs 2009, 18, 1865-1871.

20. Li, S.N.; Yang, X.W. Absorption and transportation characteristic of six linear furocoumarins in a model of Caco-2 cell monolayer in human intestine. Chin. Tradit. Herb Drugs 2011, 42, 96-102.

21. Cecchelli, R.; Berezowski, V.; Lundquist, S.; Culot, M.; Renftel, M.; Dehouck, M.P.; Fenart, L. Modelling of the blood-brain barrier in drug discovery and development. Nat. Rev. Drug Discov. 2007, 6, 650-661.

22. Abbott, N.J.; Patabendige, A.A.; Dolman, D.E.; Yusof, S.R.; Begley, D.J. Structure and function of the blood-brain barrier. Neurobiol. Dis. 2010, 37, 13-25.

23. Wang, Q.; Rager, J.D.; Weinstein, K.; Kardos, P.S.; Dobson, G.L.; Li, J.; Hidalgo, I.J. Evaluation of the MDR-MDCK cell line as a permeability screen for the blood-brain barrier. Int. J. Pharm. 2005, 288, 349-359. 
24. Hellinger, E.; Veszelka, S.; Tóth, A.E.; Walter, F.; Kittel, A.; Bakk, M.L.; Tihanyi, K.; Háda, V.; Nakagawa, S.; Duy, T.D.H.; et al. Comparison of brain capillary endothelial cell-based and epithelial (MDCK-pHaMDR, Caco-2, and VB-Caco-2) cell-based surrogate blood-brain barrier penetration models. Eur. J. Pharm. Biopharm. 2012, 82, 340-351.

25. Madgula, V.L.M.; Avula, B.; Yu, Y.B.; Wang, Y.H.; Tchantchou, F.; Fisher, S.; Luo, Y.; Khan, I.A.; Khan, S.I. Intestinal and blood-brain barrier permeability of ginkgolides and bilobalide: In vitro and in vivo approaches. Planta Med. 2010, 76, 599-606.

26. Madgula, V.L.M.; Avula, B.; Reddy, N.V.L.; Khan, I.A.; Khan, S.I. Transport of decursin and decursinol angelate across Caco-2 and MDR-MDCK cell monolayers: In vitro models for intestinal and blood-brain barrier permeability. Planta Med. 2007, 73, 330-335.

27. Pastan, I.; Gottesman, M.M.; Ueda, K.; Lovelace, E.; Rutherford, A.V.; Willingham, M.C. A retrovirus carrying an MDR1 cDNA confers multidrug resistance and polarized expression of P-glycoprotein in MDCK cells. Proc. Natl. Acad. Sci. USA 1988, 85, 4486-4490.

28. Camenisch, G.; Folkers, G.; van de Waterbeemd, H. Review of theoretical passive drug absorption models: Historical background, recent developments and limitations. Pharm. Acta. Helv. 1996, 71, 309-327.

29. Wils, P.; Warnery, A.; Phung-Ba, V.; Legrain, S.; Scherman, D. High lipophilicity decreases drug transport across intestinal epithelial cells. J. Pharmacol. Exp. Ther. 1994, 269, 654-658.

30. Yang, X.W.; Huang, X.; Ma, L.; Wu, Q.; Xu, W. The intestinal permeability of neolignans from the seeds of Myristica fragrans in the Caco-2 cell monolayer model. Planta Med. 2010, 76, $1587-1591$.

31. Zhang, C.Y.; Zhang, B.G.; Yang, X.W. Studies on the chemical constituents of the root of Angelica pubescens f. biserrata. Pharm. J. Chin. P.L.A. 2007, 23, 241-245.

32. Yang, X.W.; Zhang, C.Y.; Zhang, B.G.; Lu, Y.; Luan, J.W.; Zheng, Q.T. Novel coumarin and furan from the roots of Angelica pubescens f. biserrata. J. Asian Nat. Prod. Res. 2009, 11, 698-703.

33. Pan, L.Y.; Hu, H.H.; Wang, X.J.; Yu, L.S.; Jiang, H.D.; Chen, J.Z.; Lou, Y.; Zeng, S. Inhibitory Effects of Neochamaejasmin B on P-Glycoprotein in MDCK-hMDR1 Cells and Molecular Docking of NCB Binding in P-Glycoprotein. Molecules 2015, 20, 2931-2948.

Sample Availability: Samples are not available from the authors.

(C) 2015 by the authors; licensee MDPI, Basel, Switzerland. This article is an open access article distributed under the terms and conditions of the Creative Commons Attribution license (http://creativecommons.org/licenses/by/4.0/). 